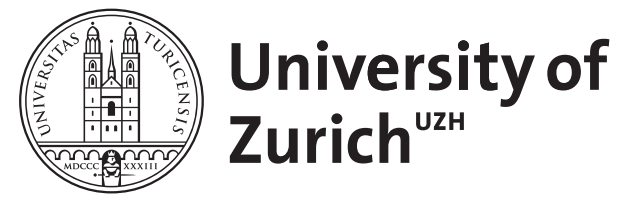

Zurich Open Repository and Archive

University of Zurich

University Library

Strickhofstrasse 39

CH-8057 Zurich

www.zora.uzh.ch

Year: 1997

\title{
Die Neuerfindung der Arbeit und die Neuordnung des Sozialstaates
}

Ruh, Hans

DOI: https://doi.org/10.14315/zee-1997-0123

Posted at the Zurich Open Repository and Archive, University of Zurich

ZORA URL: https://doi.org/10.5167/uzh-154306

Journal Article

Published Version

Originally published at:

Ruh, Hans (1997). Die Neuerfindung der Arbeit und die Neuordnung des Sozialstaates. Zeitschrift für evangelische Ethik, 41(1):162-169.

DOI: https://doi.org/10.14315/zee-1997-0123 


\section{Prospekte}

\section{Die Neuerfindung der Arbeit und die Neuordnung des Sozialstaates}

\section{Von Hans Ruh}

Ein Gesellschaftsvertrag hat die Aufgabe, die unterschiedlichen Bedürfnisse, Leistungen und Behinderungen von miteinander lebenden und kooperierenden Gruppen innerhalb einer Gesellschaft angemessen zu berücksichtigen und fair zu ordnen, und zwar unter Mithilfe des Staates. Ein wichtiger Teil eines solchen Gesellschaftsvertrages ist die Ordnung des Austausches zwischen den Generationen, eine andere Dimension betrifft das Verhältnis von Arbeitgebern und Arbeitnehmern, eine weitere das Verhältnis von Begüterten und weniger Begüterten.

Wir haben heute den Eindruck, daß die derzeit in Kraft stehenden Gesellschaftsverträge der heutigen Lage nicht mehr entsprechen. Deshalb wird mehr und mehr der Ruf nach einem neuen Gesellschaftsvertrag laut. Dieser Ruf entzündet sich heute vornehmlich im Umfeld der Arbeit. Die Arbeitslosigkeit mit all ihren Folgen läßt bisherige Regelungen als nicht mehr angemessen erscheinen. Der zentrale kritische Punkt ist wohl der, daß die Arbeitslosigkeit schwerwiegende individuelle Probleme, zum Beispiel der Identität, aber auch im Blick auf seelische Krankheiten, zugleich aber ebenso schwerwiegende soziale Probleme schafft. Weil wir die soziale Sicherheit sehr eng an die Vollbeschäftigung gekoppelt haben, gerät mit dem Ende der Arbeitsgesellschaft auch die soziale Sicherung, der Sozialstaat, in die Krise. Damit verbunden sind neue, breite Kreise umfassende Armutsformen sowie eine neue Unterschichtung der Gesellschaft.

Arbeitslosigkeit und Gefährdung der sozialen Sicherheit sind also der Anstoß für den Ruf nach einem neuen Gesellschaftsvertrag. Wie konnte es zu einer solchen Entwicklung kommen? Wie konnte es insbesondere zu den schweren Einbrüchen bei der Beschäftigung kommen ohne Aussicht auf kurz- oder mittelfristige Verbesserung, das heißt ohne Hoffnung auf die Rückkehr in die traditionale Arbeitsgesellschaft?

Es muß so etwas wie einen starken Zwang oder eine Dynamik geben, welche die Verringerung der Arbeitsplätze massiv befördert. Es muß so etwas wie einen Zwang zur Rationalisierung geben, einen Zwang zum Ersatz der menschlichen Arbeit durch technologische Mittel. Ein Element dieses Zwanges ist sicher die radikale Verschärfung der Konkurrenz in der Wirtschaft, welche durch die Globalisierung des Handels provoziert wird. Je ungehinderter die Kräfte der Konkurrenz durchschlagen, desto radikaler ist der Zwang zur Erhöhung der Produktivität und zur Verbilligung des Angebots. Diese Ziele lassen sich heute nur durch technologische Rationalisierung erreichen. Eine wesentliche Dimension dieses Vorgangs besteht darin, daß die Zeit selbst in den Zwang zur Rationalisierung hineingezogen wird. 
Die Folge ist die Erklärung der Zeit zum knappen Gut: Zeit ist Geld. Damit gerät die Zeit unter das ökonomische Gesetz: möglichst viel Produktivität in möglichst kurzer Zeit.

Diese Kombination von Konkurrenz, Ökonomisierung der Zeit und Rationalisierung wirkt auf eine ständige Verkürzung der monetarisierten Arbeitszeit und auf eine ständige Erweiterung der freien Zeit hin. Die konkrete Folge ist die Arbeitslosigkeit mit ständig steigender Tendenz. Diese Dynamik wird sich ungehindert über den ganzen Erdball fortsetzen, weil es in der globalisierten Wirtschaft keine Instanzen mehr gibt, welche dieser Markttendenz einen steuernden Rahmen beigeben können. Die Globalisierung heißt ja auch Deregulierung, das heißt wir werfen fünfzig Jahre Erfahrung der sozialen Marktwirtschaft über Bord und verzichten auf eine Marktsteuerung, beziehungsweise diese ist in der globalisierten Handelswelt gar nicht möglich. Wir haben einmal mehr das Marktgeschehen der Selbstregulation der wirtschaftlichen Kräfte überlassen, obwohl wir wissen, daß der Markt bestimmte Leistungen gar nicht erbringen kann. So ist er nicht in der Lage, ökologisch und sozial sinnvoll und gerecht zu wirken; er ist auch blind gegenüber der Sinnstiftung, vor allem aber gegenüber den Problemen der Arbeitslosigkeit. Der international frei wirkende Markt muß Arbeitslosigkeit erzeugen, denn sein Hauptkriterium ist ja die Erhöhung von Produktivität bei gleichzeitiger Verbilligung des Produkts oder der Dienstleistung.

Tatsache ist, daß die Arbeitslosigkeit gleich einem von uns inszenierten Film abläuft, in dem dauernd neue Opfer erzeugt werden, ohne daß wir diesen Film beeinflussen können. Und so hören wir gebetsmühlenartig von allen Seiten immer dieselbe Hoffnungsmelodie: Wir müssen zurück zur Vollbeschäftigung. Aber dieser Wunsch ist nun eben absolut unerfüllbar angesichts der geschilderten Dynamik des Marktes.

\section{Die Neuerfindung der Arbeit}

Die Frage ist, welche Konzepte denkbar und möglich sind zur Erreichung dieses Ziels. Wesentliche Elemente für ein solches Konzept sind die folgenden, zum Teil fundamentalen Veränderungen gegenüber den heute geltenden Konzepten:

Eine Neuaufteilung der menschlichen Tätigkeitszeit. Denkbar ist eine Dreiteilung: Freizeit, Arbeitszeit, Sozialzeit. Noch besser ist eine weitere Differenzierung in: Freizeit, monetarisierte Arbeitszeit, Eigenarbeitszeit, freiwillige Sozialzeit, obligatorische Sozialzeit, Ich-Zeit, Reproduktionszeit.

Die Förderung von sozialen, ökologischen, humanen und sinnstiftenden Leistungen durch die Förderung der freiwilligen und obligatorischen Sozialzeit.

Die Installierung eines öffentlich geförderten Arbeitsmarktes, im Sinne eines zweiten oder Komplementärmarktes.

\section{Die Neuaufteilung der menschlichen Tätigkeitszeit}

Diese Neuaufteilung der Tätigkeitszeit entsprechend den oben genannten sieben Zeiten ist eine absolute Voraussetzung für den Umbau der Arbeitsgesellschaft und des Sozialstaates. 
Nur so ist eine Flexibilisierung am Arbeitsplatz möglich. Nur so kann eine Umverteilung der Arbeit gelingen. Die Meinung ist, daß die monetarisierte Arbeitszeit sich für Männer und Frauen bei $50 \%$ des bisherigen Volumens einpendelt. Nur so ist die Förderung der Sozialzeit und der damit verbundenen Leistungen möglich. Nur so kann die Dynamik gebrochen werden, nach der immer weniger Menschen immer mehr, immer mehr Menschen immer weniger arbeiten.

\section{Die Einführung der Sozialzeit}

Die Sozialzeit zielt auf humane, soziale, ökologische Dienstleistungen, die am Markt nicht erhältlich, aber für Menschen und Gesellschaft sehr bedeutsam sind. Als Beispiele seien genannt: ökologische Landwirtschaft, Altlastensanierung, Bauschuttsanierung, Kommunikation, Pflege und Sport bei älteren Menschen, Kultur, Sicherheit in den Zügen und auf Plätzen, Arbeit mit Behinderten. Vorzusehen ist einerseits ein obligatorischer Sozialdienst, zum Beispiel von einem Jahr in der Jugend, als Wiederholungskurse durch das Leben, als Blöcke nach der Pensionierung. Andererseits soll die Freiwilligenarbeit gefördert werden.

Die Realisierung der Sozialzeit ist von hoher Bedeutung für die Neuverteilung der Tätigkeiten, für die Sicherstellung von gesellschaftlich bedeutsamen Leistungen, insbesondere für die nicht monetäre Sicherstellung von sozialstaatlichen Leistungen bei Lebensrisiken und im Alter. Weil die genannten Leistungen über den normalen Arbeitsmarkt nicht erhältlich sind, braucht es die Realisierung der Sozialzeit und zwar sowohl in Form eines obligatorischen Sozialdienstes wie in Form einer umfassenden Förderung und Organisation freiwilliger Dienste. Die Struktur des obligatorischen Sozialdienstes ist in manchen Ländern in Form des Militärdienstes bekannt. Er ist das notwendige Gegenstück zum Grundlohn.

Die Freiwilligenarbeit scheint sich immer mehr durchzusetzen. Schon arbeiten in den USA über 80 Millionen Amerikanerinnen und Amerikaner mehr als fünf Stunden wöchentlich freiwillig. In anderen Ländern wächst die Freiwilligenbewegung an und immer neue Formen des Einsatzes und der Koordination sind im Entstehen. Auf jeden Fall ist die Akzeptanz der Sozialzeit deutlich im Steigen begriffen.

\section{Die Installierung eines öffentlich geförderten Komplementärmarktes}

Die bisher genannten Elemente sind die notwendigen Voraussetzungen für die Schaffung eines zweiten Arbeitsmarktes. Dieser ist notwendig, weil im normalen Arbeitsmarkt zu wenig Arbeit vorhanden ist und weil gleichzeitig gesellschaftlich bedeutsame und notwendige Leistungen nicht erhältlich sind. Der zweite Arbeitsmarkt ist die Antwort auf das Faktum, daß die Welt voller ungelöster Probleme, also voller Arbeit ist, die Menschen aber daran gehindert werden, ihre Gaben und Kräfte für die Lösung dieser Probleme einzusetzen. Der zweite Arbeitsmarkt funktioniert projektbezogen. Die Kommunen sind dabei federführend, indem sie Projekte ausschreiben und fördern. Finanziert werden solche Projekte von Industrien, Kommunen, privaten und gemeinnützigen Gesellschaften. Beispiels- 
weise wird ein großes Unternehmen, das Arbeitsplätze rationalisiert und dabei Gewinne macht, einen bestimmten Betrag zur Förderung solcher Projekte einsetzen. Sofern solche Projekte langfristige Rendite versprechen, zum Beispiel im Energie- und Ökologiebereich, werden Banken um langfristige Kredite angegangen.

Neben der Ermöglichung von notwendigen Dienstleistungen hat der zweite Arbeitsmarkt einen kostensenkenden Effekt für die gesellschaftlichen beziehungsweise volkswirtschaftlichen Fixkosten. Er senkt zum Beispiel die Umweltkosten, welche vermutlich 300 bis 400 Milliarden DM pro Jahr in Deutschland erreichen. Er senkt die Gesundheitskosten. Dazu nur eine Zahl: Wenn in Deutschland viele Milliarden Krankheitskosten und Arbeitsverlustkosten anfallen wegen arbeitsbedingten Rückenschäden, dann sinken diese Kosten im Rahmen eines neuen Arbeitszeitmodells und einer neuen Zeiteinteilung beträchtlich. Weiter werden die Gewaltkosten gesenkt, vor allem aber die ins Unermeßliche gestiegenen Kosten für die seelischen Folgen der Arbeitslosigkeit.

Wer arbeitet nun in Projekten des zweiten Arbeitsmarktes? Dies sind natürlich Arbeitslose, aber auch Frührentner, freiwillig Arbeitende, obligatorisch Dienstleistende, Behinderte und einige professionelle Spezialisten. Eine Idee dabei ist, daß auf reine Arbeitsloseneinsätze verzichtet wird und daß stets gemischte Gruppen angesprochen werden. Mit einem solchen Konzept wird nicht nur erreicht, daß fast alle Menschen beschäftigt sind, es werden auch die Gaben und Tätigkeiten derjenigen genutzt, die diese der Gesellschaft weiter zur Verfügung stellen wollen oder die im Arbeitsmarkt nicht zum Zuge kommen. Ein besonderes Gewicht wird auf die Entwicklung von neuen Technologien gelegt.

Die berechtigte Hoffnung besteht, daß die Existenz eines solchen Komplementärmarktes mit der Zeit die Präferenzen für den Wert der Arbeit verändern werden. Menschen werden die Erfahrung machen, daß humane und ökologische Dienstleistungen einen sinnstiftenden Charakter haben. Es könnte wohl sein, daß damit bestimmte Dienstleistungen vom zweiten in den ersten Arbeitsmarkt zurückwandern, was durchaus zu begrüßen ist. Prinzipiell wird allerdings darauf zu achten sein, daß der zweite Arbeitsmarkt keine unfaire Konkurrenz zum ersten Arbeitsmarkt darstellt, was auf Grund der Beantwortung der Frage »Welche Leistungen erbringt der Markt, welche nicht? « einigermaßen zu klären ist.

Einen besonderen Anreiz für den zweiten Arbeitsmarkt soll ein umfassendes und qualitativ hochstehendes Bildungsangebot darstellen. Dabei geht es um die Förderung von personalen und sozialen Kompetenzen, dann auch um fachliche Weiterbildung. Dieses Bildungsangebot muß höchst attraktiv sein, wird aber kostengünstig zu gestalten sein, wenn auch hier die Tätigkeiten der Frühpensionierten, der Freiwilligen und anderer Gruppen eingesetzt werden.

Diese Überlegungen zum zweiten Arbeitsmarkt basieren nicht zuletzt auf der Überzeugung, daß die Aktivierung von Millionen von Menschen sich positiv auswirken muß auf die gesellschaftlichen Fixkosten wie vor allem auf die Lebensqualität einer Gesellschaft. Diese Überlegungen bekämpfen endlich eine Voraussetzung der heutigen Entwicklung, nämlich daß Geldverdienen und Konkurrenz zu den höchsten Gütern auf dieser Welt zählten. Wir können nur sinnvoll weiterleben, wenn wir diese Ziele in ihrer Bedeutung massiv herabzustufen bereit sind.

Die Konzeption des öffentlich geförderten Arbeitsmarktes muß insbesondere den folgenden Kriterien genügen: 
- Es dürfen keine falschen und überholten Strukturen erhalten werden

- Die Leistungen dieses Marktes müssen gesellschaftlich notwendig sein und über den 1. Arbeitsmarkt nicht oder unvollkommen erhältlich sein

- Die Fixkosten der Gesellschaft müssen durch den 2. Arbeitsmarkt gesenkt werden, zum Beispiel Umwelt-, Sicherheits-, Arbeitsplatz- und Gesundheitskosten

- Es darf keine öffentlich geförderte Konkurrenz zum 1. Arbeitsmarkt geben; hingegen sind Teile des 1. Arbeitsmarktes am 2. Arbeitsmarkt angemessen zu beteiligen

- Es dürfen keine Strategien verfolgt werden, die langfristig falsch, zum Beispiel ökologisch falsch sind

- Im 2. Arbeitsmarkt muß ein hoher Anteil an Bildung vermittelt werden, zum Teil über freiwillige Dienstleistungen.

\section{Strategien zur sozialstaatlichen Sicherheit}

Die bisher vorgestellte Umverteilung der Arbeit, die Neuordnung der Zeiteinteilung, das Konzept der Sozialzeit sowie das Konzept eines öffentlich geförderten Arbeitsmarktes sind notwendige Elemente eines neuen Gesellschaftsvertrages. Diese Elemente sind nun unter dem Gesichtspunkt einer neuen Konzeption des Sozialstaates zu reflektieren. Der Sozialstaat gilt ja nicht bloß als nicht mehr bezahlbar, er ist auch in seiner legitimatorischen Begründung gefährdet. Allenfalls soll noch gelten, daß der Staat den am wenigsten Privilegierten Sozialhilfe nach dem Bedürfnisprinzip leistet. Im Zusammenhang mit dem Sozialstaat gibt es eigentlich zwei zentrale Probleme. Einerseits ist die Legitimation des Sozialstaates in eine Krise geraten, andererseits werden Zweifel an der ökonomischen Machbarkeit des bisherigen Sozialstaates laut, vermischt auch mit dem Verdacht, daß der Sozialstaat die Leistungsbereitschaft senkt und falsche Ausnützungstendenzen fördert.

Zuerst zur Krise der Legitimation. In der europäischen Tradition galten die sozialen Menschenrechte immer weniger als die individuellen Menschenrechte. So galten und gelten letztere als besser implementierbar als die sozialen Rechte. Insbesondere wirkt sich aus, daß sowohl in der Tradition von Adam Smith als auch in der Tradition von Immanuel Kant letztlich der Glaube an die moralische Selbstorganisation des wirtschaftlichen System, letztlich an die unsichtbare Hand, besteht. Dieser Glaube relativiert den sozialstaatlichen Handlungsbedarf. Hinzu kommen nun noch einige andere Elemente: So ist der Staat angewiesen auf die Zustimmung der Mehrheit; diese ist aber nur für minimale Relegungen und eher für Maßnahmen zu haben, welche allen Vorteile bringen, wie zum Beispiel der StraBenbau. Endlich besteht immer die Tendenz, daß die wirtschaftlich Starken auf möglichst wenig Regelungen drängen, weil die Starken bei weniger Regelungen Vorteile gegenüber den Schwachen haben. Die europäische Tradition verhält sich also immer wieder reserviert gegenüber der Idee des Sozialstaates, im Unterschied etwa zum politischen System, in dem ein demokratischer Regelungsbedarf unbestritten ist.

In diesen ganzen Zusammenhang gehört die Feststellung, daß die Entwicklung der Akzeptanz der Solidaritätsidee ambivalent ist und widersprüchlich verläuft. Auf der einen Seite gibt es eine neue Bereitschaft zum freiwilligen sozialen Engagement, auf der anderen 
Seite ist ein Zerfall der Solidaritätsidee zu beobachten. Noch immer ist diese Solidaritätsidee sehr stark religiös oder ethisch begründet, insbesondere von der historischen Entwicklung her gesehen. Aber gerade deshalb wird mehr und mehr die Frage laut, warum ein Staat eine religiöse oder ethische Idee zwangsweise durchsetzen soll, welche mit großen Einschränkungen an Freiheit und Leistungen an Geld verbunden ist.

Die Frage stellt sich, ob nicht nach einer neuen, umfassenderen Begründung und Legitimierung des Sozialstaates zu suchen ist. Mir scheint, diese Frage ist zu bejahen. In welcher Richtung ist sie aber zu beantworten?

1. In der modernen Gesellschaft können die wichtigsten Aufgaben nur kooperativ-solidarisch erfültt werden. Die Existenz wichtiger gesellschaftlicher und wirtschaftlicher Institutionen setzt die solidarische Kooperation sehr vieler und unterschiedlicher gesellschaftlicher Gruppen voraus. Diese Behauptung bezieht sich auf die äußere Sicherheit, also die Institution Armee, die innere Sicherheit, die Rechtssicherheit, die Wirtschaft, die Bildung. Alle diese Aufgaben sind nur als kooperativ-solidarische Verrichtung denkbar.

2. Diese solidarische Kooperation verläuft als permanenter Austausch oder Tauschvorgang zwischen den unterschiedlichsten Gruppen und Menschen. Dabei zeichnet sich dieser Tauschvorgang aus durch eine Ungleichheit hinsichtlich der Verteilung von Vor- und Nachteilen, Leistungen und Beanspruchungen, Verzichten und Anstrengungen, Bedürfnissen und Notständen.

Anhand einiger Beispiele sollen solche ungleiche Austauschprozesse konkret dargestellt werden. Die einen, vor allem Besitzende, profitieren von Anstrengungen für die innere und äußere Sicherheit, andere, zum Beispiel Soldaten und Polizisten, gehen dafür Risiken ein. Die einen wohnen an einer lärmenden Autobahn, andere profitieren von einer ruhigen Wohnlage und genießen außerdem überproportional die Güter und Dienstleistungen, die über die Autobahn herangefahren werden. Die einen schonen die Umwelt durch Verzichtleistungen, andere belasten die Umwelt, haben dafür Vorteile, deren Nachteile aber für alle gleich sind. Bestimmten Gruppen werden Verzichtleistungen abgefordert, zum Beispiel den körperlich Starken, während wirtschaftlich und intellektuell Starke von Eigentumsrechten geschützt werden.

Die wirtschaftliche und gesellschaftliche Kooperation verläuft weitgehend in Form von ungleichen Austauschprozessen. Dabei gibt es Gewinner und Verlierer, wobei eine exakte Identifikation und Quantifizierung unmöglich ist. Eindeutig und für unsere Frage entscheidend ist, daß durch diese Austauschprozesse Reiche und Arme, unterschiedliche Schichtungen der Gesellschaft geschaffen werden.

3. Ganz entscheidend ist nun, daß der Staat an diesen ungleichen Austauschprozessen ursächlich, direkt und indirekt, beteiligt ist. So fördert die Rechtsprechung, zum Beispiel die Durchsetzung der Eigentumsgarantie, solche ungleiche Austauschprozesse. Die Verkehrspolitik schafft Vorteile und Belastungen. Internationale Abkommen schaffen soziale Ungleichheit und Arbeitslosigkeit. Der Staat ist also eminent an den genannten Tauschvorgängen beteiligt, nicht zuletzt durch seine Bildungspolitik. Er setzt Rahmenbedingungen für solche Tauschvorgänge mit staatlicher Gewalt durch. Er ist also auch beteiligt an der Herstellung von sozialer Ungleichheit, Arbeitslosigkeit, Umweltverschmutzung. 
Schon hier ist darauf aufmerksam zu machen, daß es nicht zunächst um eine Kritik an diesen Tauschvorgängen und der staatlichen Beteiligung daran geht. Dies alles ist, zumindest weitgehend, unvermeidlich. Der springende Punkt ist der, daß der Staat mit staatlichen Mitteln an der Durchsetzung dieser Vorgänge beteiligt ist.

4. Mitunter wirkt die staatliche Beteiligung an diesen Austauschprozessen in der Weise, $\mathrm{da} ß$ sie die Verantwortung, die Autonomie und den Spielraum des einzelnen einschränkt. So ist Arbeitslosigkeit oder Sozialhilfebedürftigkeit sehr oft kein individuell verschuldetes Schicksal, sondern die Folge struktureller Bedingungen, die zum Teil vom Staat durchgesetzt werden.

Die internationale Arbeitsteilung fördert Arbeitslosigkeit. Die Verkehrspolitik beeinträchtigt Gesundheit und Wohlbefinden, Beeinträchtigungen, denen sich der einzelne oft nicht entziehen kann. Die Landwirtschaftspolitik lenkt das Handeln der Bauern in eine gewisse Richtung. Man kann das allgemein so ausdrücken: Der Ausdifferenzierungsprozess der Gesellschaft engt den Handlungsspielraum des einzelnen mehr und mehr ein.

5. Das Fazit dieser thesenartigen Überlegungen lautet: Die gesellschaftliche solidarische Kooperation, die ungleichen Austauschprozesse unter Beteiligung des Staates rufen nach einer verstärkten Durchsetzung von Korrekturen, insbesondere sozialen Korrekturen, durch den Staat. Dabei ist zu beachten, daß zwar die Austauschprozesse ungleich sind, die Ungleichheit aber schwer zu quantifizieren und zu identifizieren ist. Weiter: Die ökonomisch-gesellschaftliche Arbeitsteilung, an der der Staat beteiligt ist, macht immer mehr Menschen abhängig und schränkt deren Handlungsspielraum ein. Oder anders gesagt: Viele gesellschaftliche Ungleichheiten sind staatliche Konstruktionen und müssen durch andere staatliche Konstruktionen korrigiert werden.

Aber nun stellt sich die Frage nach dem »Wie«. Zentral muß der Gedanke sein, daß, angesichts der Schwierigkeit einer Quantifizierung die staatliche Korrektur pragmatisch und in gewisser Weise pauschalierend erfolgen muß. Von dieser Überlegung her erscheint es zwingend, daß wir die soziale Sicherheit einmal als eine zentrale Aufgabe des Staates verstehen, wie die anderen genannten Aufgaben auch, daß aber wegen der geforderten Pauschalisierung am ehesten eine arbeitsunabhängige Grundsicherung für alle in Frage kommt.

\section{Teilweise Entkoppelung von Arbeit und Lohn}

Es gibt eine Menge von Gründen, die für eine solche Entkoppelung sprechen. Zunächst muß eine Gesellschaft, welche durch strukturelle Arbeitslosigkeit und Niedriglohnpolitik die Menschen an der Sicherung des Lebensunterhalts hindert, einen arbeitsunabhängigen Grundlohn, eine Bürgerrente für alle, ausbezahlen. Es gibt aber auch ethische Gründe für die Bürgerrente: Die positiven oder negativen Leistungen der Menschen lassen sich nicht vollständig identifizieren, weder innerbetrieblich noch gar gesellschaftlich. Der Saldo der Leistungen geht nicht, auf jeden Fall nicht vollständig, entlang der Lohnskala. Weiter kommt dazu, daß Fähigkeiten und Verschuldungen von Menschen nur zum Teil in der Verantwortung des einzelnen liegen. Vieles hängt von Umständen, zum Beispiel Elternhaus und Gesundheit ab. 
Es gibt also viele Gründe dafür, einen Sockelbetrag als Bürgerrente oder Grundlohn für alle vorzusehen. Hier wird an die Größenordnung von DM 1500,-- pro Monat und Person gedacht. Dies ist nicht existenzsichernd. Gedacht ist, daß im Normalfall die Menschen dazu noch während circa 50\% der bisherigen Arbeitszeit arbeiten. Der Grundlohn hat die Bedeutung, da $ß$ damit eine Grundsicherung für alle sowie die Voraussetzung für die Flexibilisierung am Arbeitsplatz und für die Sozialzeit geschaffen wird. Der Grundlohn ist so unabdingbare Voraussetzung für eine Umverteilung der Arbeit, für die Sicherstellung notwendiger Dienstleistungen und für eine teilweise arbeitsunabhängige Sicherung der Lebensrisiken.

Die finanzielle und ökonomische Sicherstellung des Grundlohnes erfolgt auf drei Ebenen: durch eine ökologische Steuerreform, durch die präventive Einsparung von Kosten, durch die Leistungen in der Sozialzeit.

Und endlich: Der Grundlohn ist eine Antwort auf den Skandal, daß eine Gesellschaft sich eine luxuriöse Zivilisation für wenige leistet, gleichzeitig aber die Verarmung breiter Schichten in Kauf nimmt.

Prof. Dr. Hans Ruh

Ethik-Zentrum der Universität Zürich

Institut für Sozialethik

Zollikerstraße 117

CH-8008 Zürich 